\title{
Growth and puberty after growth hormone treatment after irradiation for brain tumours
}

\author{
A L Ogilvy-Stuart, S M Shalet
}

\begin{abstract}
The impact of treatment with either cranial or craniospinal irradiation with or without cytotoxic chemotherapy for a brain tumour distant from the hypothalamic-pituitary axis was assessed in 29 children who had reached final height. All had received growth hormone treatment for radiation induced growth hormone deficiency. Final height, segmental growth during puberty, and duration of puberty were studied.

Both craniospinal irradiation and the use of chemotherapy resulted in a significant and equal reduction in final height; this effect in those children who received both craniospinal irradiation and chemotherapy was additive. The degree of height loss was related to the age at irradiation, the most profound effect on final height occurring in the youngest at irradiation. The mean duration of puberty from G2-G4/B2-B4 (1.97 years) was not significantly different from the duration of puberty in normal children.

Growth hormone increases growth velocity in children with radiation induced growth hormone deficiency but their final height is significantly less than their mid-parental height. The use of spinal irradiation and chemotherapy in the original treatment of brain tumours has a marked effect on growth which is not overcome with the use of growth hormone treatment in current doses. Early puberty of normal duration contributes to poor growth.
\end{abstract}

(Arch Dis Child 1995; 73: 141-146)

Keywords: brain tumour, growth hormone treatment, chemotherapy, final height.

For a number of years it has been realised that treatment of intracranial malignancy with cranial irradiation of $27 \mathrm{~Gy}$ or more to the hypothalamic-pituitary axis resulted in growth hormone deficiency and growth impairment. ${ }^{12}$ In addition to growth hormone deficiency, a number of factors were identified that may contribute to poor growth, including spinal irradiation, inadequate nutrition, adjuvant cytotoxic chemotherapy, and tumour recurrence.

A number of early short term studies reported an improved growth response to growth hormone treatment in children with radiation induced growth hormone deficiency. ${ }^{3-5}$ More recently, several studies have distinguished between those receiving cranial and craniospinal irradiation protocols, ${ }^{6-9}$ and demonstrated the marked detrimental effect of spinal irradiation on subsequent spinal growth that was not ameliorated by growth hormone treatment. Final height studies on small numbers of children treated with growth hormone for radiation induced growth hormone deficiency indicate it failed to induce catch-up growth, but prevented further height loss. ${ }^{6-8}$

Children who have received cranial irradiation with a dose in excess of 25 Gy are vulnerable to early puberty, the age of onset of puberty being related to the age at irradiation. ${ }^{10}$ The duration of puberty in these patients is unknown, but it has been suggested that in children with idiopathic growth hormone deficiency, treated with growth hormone treatment, puberty is attenuated. ${ }^{11}$

The effect of chemotherapy on the growth of children treated for brain tumours has not been studied in detail, but there is a suggestion that chemotherapy affects subsequent growth adversely. ${ }^{12}$

The aim of this study was to assess final height in children treated with growth hormone after treatment of a brain tumour distant from the hypothalamic-pituitary axis, and to study the individual impact of the initial treatment modalities, that is, cranial or craniospinal irradiation, and the impact of chemotherapy on subsequent growth. Final height in relation to mid-parental height, segmental growth during puberty, and the duration of puberty were also determined.

\section{Patients and methods PATIENTS}

The patient population consisted of all 47 children who had received at least one year of growth hormone treatment for irradiation induced growth hormone deficiency after treatment of a brain tumour distant from the hypothalamic-pituitary axis at the Christie Hospital, Manchester between 1975 and 1991.

Thirteen children with a histological diagnosis of a locally invasive tumour received a single fractionated course of radiotherapy to the cranium only. The hypothalamic-pituitary axis was within the radical radiotherapy treatment volume. In these children, the median dose of irradiation was 42.5 Gy (range 29.5-47.5 Gy) administered in 16 (range 8-21) fractions over 21 (9-28) days.

Children with a tumour with the propensity to disseminate throughout the central nervous system received an initial fractionated course of radiotherapy to the tumour site, which in most cases was the posterior fossa. The hypothalamic-pituitary fossa is located at the 
Table 1 Tumour diagnosis, sex, and treatment protocols in the 47 children treated for at least one year with growth hormone for radiation induced growth hormone deficiency

\begin{tabular}{lccccc}
\hline Diagnosis & No (\%) & $\begin{array}{l}\text { Craniospinal } \\
\text { irradiation }\end{array}$ & $\begin{array}{l}\text { Cranial } \\
\text { irradiation }\end{array}$ & Chemotherapy & Male \\
\hline Medulloblastoma & $27(57 \cdot 4)$ & 27 & 0 & 9 & 16 \\
Ependymoma & $5(10 \cdot 6)$ & 4 & 1 & 3 & 4 \\
Juvenile astrocytoma & $7(14 \cdot 9)$ & 1 & 6 & 2 & 4 \\
Adult astrocytoma & $4(8 \cdot 5)$ & 1 & 3 & 0 & 1 \\
Other glioma & $4(8 \cdot 5)$ & 1 & 3 & 1 & 2 \\
Total & 47 & 34 & 13 & 15 & 27 \\
\hline
\end{tabular}

edge of this radiation field. The median (range) dose of irradiation to the tumour site was $15(10-20)$ Gy in 10 (4-16) fractions over 11 (5-18) days. This is followed by a course of radiotherapy to the head, neck, thoracic, and lumbar spine. The median dose of craniospinal irradiation was $30(15-35) \mathrm{Gy}$ in 20 (15-24) fractions over 27 (13-45) days. Children treated before 1972 received multiple field irradiation, including the spine, without a separate dose to the tumour site. In most children the spinal irradiation is given as oblique wedge pairs, but in very young children (less than 2 years of age) this is given as a single posterior field with a reduced dose. Thirty four children received craniospinal irradiation.

In the treatment of the original disease, 18 children (13 of those receiving craniospinal irradiation) received 12 to 18 months of chemotherapy which consisted of vincristine alone $\left(1.5 \mathrm{mg} / \mathrm{m}^{2}\right)$ or in combination with carmustine (BiCNU) $\left(100 \mathrm{mg} / \mathrm{m}^{2}\right)$, or BiCNU $\left(100 \mathrm{mg} / \mathrm{m}^{2}\right)$ alone, given every six weeks for a period of 12 months (Manchester protocol before 1975); vincristine, $1.5 \mathrm{mg} / \mathrm{m}^{2}$, in combination with lomustine (CCNU), $40 \mathrm{mg} / \mathrm{m}^{2}$, or procarbazine, $100 \mathrm{mg} / \mathrm{m}^{2}$, or both given during a period of 18 months (UK Children's Cancer Study Group protocol after 1979). One child received two courses of mustine, vincristine, prednisolone, and procarbazine (MOPP), and one child, cisplatin.

The patient characteristics (tumour diagnosis, sex, treatment protocols) are shown in table 1 .

In addition to growth hormone deficiency, four had a deficiency of other anterior pituitary hormones (two gonadotrophin deficient, and two thyrotrophin deficient) which were treated with appropriate hormone replacement. Sixteen children had raised gonadotrophin concentrations indicative of gonadal damage secondary to chemotherapy, or radiation scatter from the spinal field of craniospinal irradiation. Four of the 16 (all girls) required exogenous oestrogen for induction of puberty. The growth data from these four were excluded from the puberty studies. Seven children had a raised concentration of thyroid stimulating hormone (TSH) in the presence of a normal $(n=5)$, or a low $(n=2)$ thyroxine concentration. All seven were treated with thyroxine to return the TSH concentration into the normal range.

In all children, growth hormone deficiency (peak growth hormone concentration $<20$ $\mathrm{mU} / \mathrm{l}(<10 \mu \mathrm{g} / \mathrm{l})$ ) was confirmed on provocative testing with arginine stimulation and either insulin induced hypoglycaemia $(0.2 \mathrm{U} / \mathrm{kg}$ human soluble insulin intravenously to produce a blood glucose nadir of $<2.2 \mathrm{mmol} / \mathrm{l}$ ), or a glucagon stimulation test $(15 \mu \mathrm{g} / \mathrm{kg}$, to a maximum of $1 \mathrm{mg}$ ).

Before May 1985, children received $5 \mathrm{mg}$ of pituitary derived growth hormone three times a week by intramuscular injection. Thereafter synthetic growth hormone was used, initially at a dose of 4 IU three times a week irrespective of weight. Since 1988 children have received $0.5 \mathrm{U} / \mathrm{kg} /$ week, administered by daily subcutaneous injection. The median duration of growth hormone treatment in the 29 children who have reached final height was 3.7 years (range 1.3-7.5 years). The median age at the start of growth hormone in these children was $11 \cdot 4$ years (range $7 \cdot 4-16 \cdot 0$ years).

\section{AUXOLOGY}

Anthropometry was carried out every three to four months on wall and table mounted stadiometers by trained observers to determine standing and sitting height measurements. Subischial leg length measurements have been extrapolated from these direct measurements. Pubertal status was assessed by the method of Marshall and Tanner. ${ }^{1314}$ Testicular volumes were estimated by comparison with the Prader orchidometer. ${ }^{15}$ Bone age was assessed every six to 12 months by the TW2 method. ${ }^{16}$

The raw data have been transformed to SD scores by the formula:

SD score $=$

observed measurement $(\mathrm{cm})-$ population mean for age and sex $(\mathrm{cm})$ SD around that mean

Values for the population means and SD are taken from the data of Tanner and Whitehouse. ${ }^{17}$

Final height has been defined as that height achieved when measurements over 12 months differ by less than $1 \mathrm{~cm}$.

\section{METHODS}

The efficacy of growth hormone was assessed by comparing leg length growth velocity after the first year of treatment with pretreatment leg length growth velocity in all 19 children who remained prepubertal at the end of the first year of growth hormone treatment. In this way the efficacy of growth hormone can be assessed irrespective of the influence of radiation on spinal growth and without the influence of sex steroids.

Twenty nine children (17 boys) have completed their growth and the impact of the different treatment modalities on final height assessed. Final height in relation to midparental height has been assessed in 24 of these 29 children from measured $(n=5)$ or reported $(n=19)$ parental heights.

In 18 of the 29 in whom onset of puberty data were available, segmental growth (that is changes in sitting height and leg length) during puberty has been studied. 


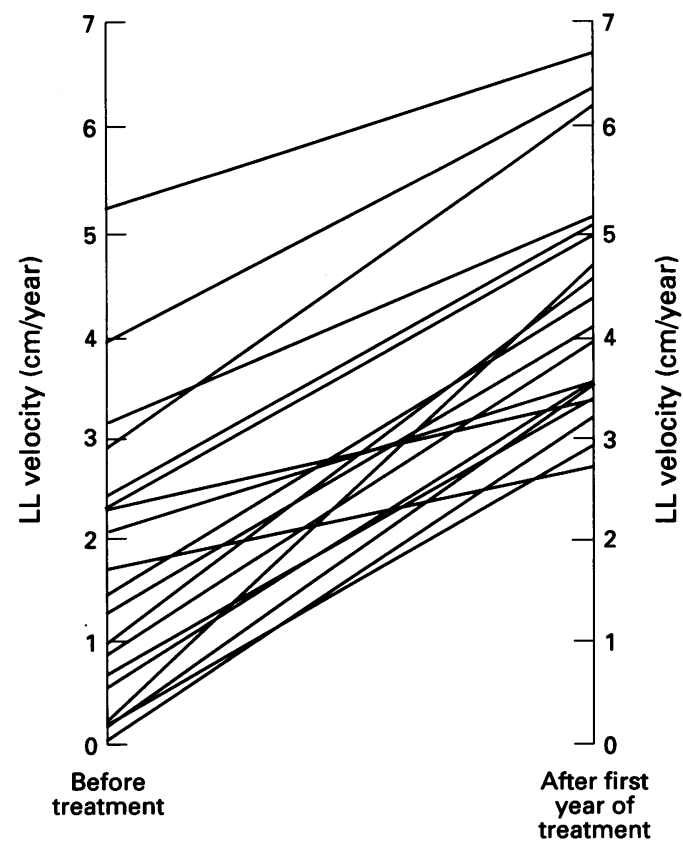

Figure 1 Subischial leg length (LL) velocities before and after first year of growth hormone treatment.

Duration of puberty, that is time from the onset of puberty (G2 or B2) to stage G4 or B4 was studied in 26 children in whom these data were available. The impact of the different treatment modalities on length of puberty was assessed. The age at pubertal onset was taken as the mid-age between clinic visits when the child was last noted to be prepubertal (that is G1 or B1) and the next when he/she was G2 or B2 (mean (SE) interval between B1 and B2 or G1 and G2 clinic visits, $0.37(0.04)$ years). Similarly, the age at G4/B4 was taken as the mid-age between clinic visits when the child was G3/B3 and the next when he/she had attained pubertal stage G4/B4 (mean (SE) interval between these clinic visits $0.33(0.03)$ years).

The mean (SE) age of onset of spontaneous puberty in 30 of the whole group of 47 children on whom data were available was $10.9(0.35)$ years in the boys and $9.9(0.49)$ years in the girls. The segmental measurements at the onset of puberty were extrapolated linearly from the differences in measurements at clinic visits before and after the onset of puberty.

Disproportion at final height has been calculated by the formula: (LL-SH) $-\left(\mu_{L L}-\mu_{S H}\right)$ where $\mu$ is the population mean; LL=leg length, $\mathrm{SH}=$ sitting height. The 29 children who had reached final height were cross classified by type of irradiation (that is cranial or craniospinal irradiation) and whether or not chemotherapy had been used. As there were unequal numbers in the four groups a regression approach to the analysis was used, employing appropriate dummy variables.

\section{STATISTICS}

Regression analysis was applied to estimate the impact on final height and on segmental growth of the different radiotherapy protocols (that is cranial $v$ craniospinal irradiation) and chemotherapy after adjusting for possible

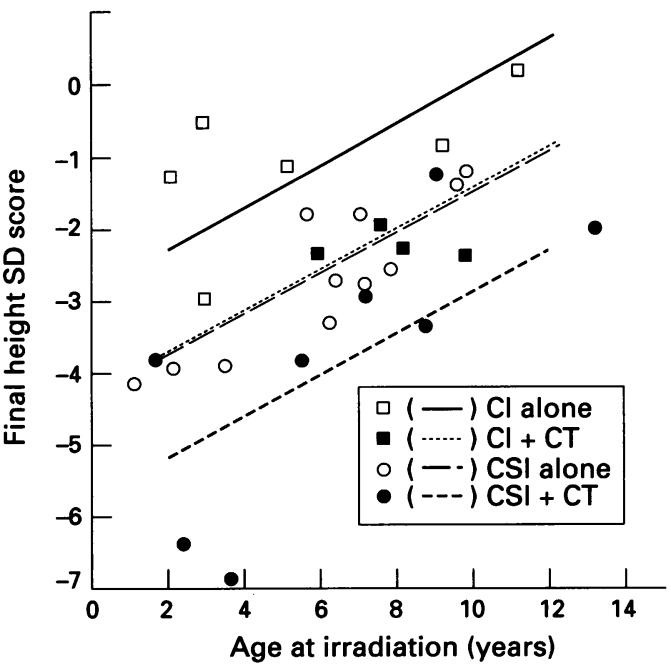

Figure 2 Parallel linear regressions of final height SD score on age at irradiation according to the four treatment groups: cranial irradiation (CI) alone, $C I$ plus chemotherapy (CT), cranial irradiation alone (CSI), and CSI plus $C T$.

effects of the lag time between irradiation and the start of growth hormone treatment and the age at irradiation. As the lag time was strongly negatively correlated with the age at irradiation and did not contribute additional information, it was omitted from the model.

The duration of puberty was positively skewed in the 26 children studied, therefore the data were log transformed and analysis of variance (two factor ANOVA) was used to assess the impact of the treatment modalities.

\section{Results}

GROWTH AFTER START OF GROWTH HORMONE In all cases there was an increase in leg length velocity after the start of growth hormone treatment (fig 1). In all except one case the leg length velocity after one year of growth hormone treatment was at least $1 \mathrm{~cm}$ greater than the pretreatment velocity. The mean (SE) pretreatment leg length velocity was $2 \cdot 0(0 \cdot 3)$ $\mathrm{cm} /$ year and after treatment $4.3 \mathrm{~cm} /$ year $(\mathrm{SE}$ $0 \cdot 28$ ).

\section{FINAL HEIGHT}

Both craniospinal irradiation and the use of cytotoxic chemotherapy resulted in a significant and equal reduction in final height in children treated with growth hormone for radiation induced growth hormone deficiency. The effect on final height in those children who received both craniospinal irradiation and chemotherapy was additive, there being no interaction of irradiation and chemotherapy. The degree of height loss was related to the age at irradiation, and may be a product of the longer lag time in the younger children before the start of growth hormone treatment.

The parallel linear regressions of final height SD score on age at irradiation according to the four treatment groups, cranial irradiation alone, cranial irradiation plus chemotherapy, craniospinal irradiation alone, craniospinal irradiation plus chemotherapy, are shown in fig 2 . Note the 
Table 2 Difference between mid-parental height and final height achieved

\begin{tabular}{llcl}
\hline Treatment group & $\begin{array}{l}\text { No of cases } \\
(n=24)\end{array}$ & $\begin{array}{l}\text { Mean }(S D) \text { height } \\
\text { difference }(\mathrm{cm})\end{array}$ & $\begin{array}{l}\text { Mid-parental } \\
\text { height }(\mathrm{cm})\end{array}$ \\
\hline Cranial irradiation & 6 & $7 \cdot 2(6 \cdot 7)$ & $171 \cdot 3$ \\
Cranial irradiation+chemotherapy & 3 & $18 \cdot 4(3 \cdot 5)$ & $174 \cdot 9$ \\
Craniospinal irradiation & 9 & $17 \cdot 0(5 \cdot 9)$ & $169 \cdot 2$ \\
Craniospinal irradiation+chemotherapy & 6 & $24 \cdot 5(11 \cdot 1)$ & $171 \cdot 3$ \\
\hline
\end{tabular}

regression line is the same for cranial irradiation plus chemotherapy and craniospinal irradiation alone and that the differences between the regression lines are equal. Thus the estimated loss in standing height despite treatment with growth hormone in a child treated with either craniospinal irradiation alone or cranial irradiation and chemotherapy would be approximately $9.5 \mathrm{~cm}$ more at each age at irradiation than in a child who had cranial irradiation alone and in a child who received both craniospinal irradiation and chemotherapy this loss would amount to $19 \mathrm{~cm}$ compared with a child who received cranial irradiation alone. In clinical terms therefore, a child who received either craniospinal irradiation without chemotherapy, or cranial irradiation with chemotherapy at the age of 2 years, will be approximately $24.7 \mathrm{~cm}$ shorter at final height than a child on the 50th centile, and a child receiving both craniospinal irradiation and chemotherapy at 2 years of age will be approximately $34.2 \mathrm{~cm}$ shorter than a child on the 50 th centile at final height. The $95 \%$ confidence interval for the reduction in final height SD score due to either craniospinal irradiation or chemotherapy is 0.96 to 1.98 .

SEGMENTAL PROPORTIONS AT FINAL HEIGHT There was no significant effect of either chemotherapy alone $(p=0.47)$, or synergistically with cranial irradiation $(p=0.61)$ on skeletal disproportion. However, at final height, the children who received craniospinal irradiation were significantly disproportionate $(p<0.0001)$ with short backs and long legs. The use of chemotherapy did not influence proportion in these patients.

\section{PARENTAL HEIGHT}

The difference between mid-parental height and final height achieved for the four treatment groups is shown in table 2 . One child (who received cranial irradiation alone at $11 \cdot 0$

Table 3 Duration of puberty (G2 or B2 to G4 or B4) in the four treatment groups

\begin{tabular}{lcl}
\hline Treatment group & No of cases & $\begin{array}{l}\text { Mean (SD) duration } \\
\text { puberty (years) }\end{array}$ \\
\hline Cranial irradiation & 3 & $2.53(0.27)$ \\
Cranial irradiation+chemotherapy & 2 & $1 \cdot 77(0.23)$ \\
Craniospinal irradiation & 12 & $1.82(0.43)$ \\
Craniospinal irradiation+chemotherapy & 9 & $2.06(0.44)$ \\
Whole group & 26 & $1.97(0.40)$ \\
\hline
\end{tabular}

Table 4 Disproportion at final height (cm); for calculation see Methods section

\begin{tabular}{lcc}
\hline Treatment group & No of cases & Mean $(S E)(\mathrm{cm})$ \\
\hline Cranial irradiation & 4 & $-0 \cdot 13(1.91)$ \\
Cranial irradiation +chemotherapy & 4 & $-2 \cdot 88(3.41)$ \\
Craniospinal irradiation & 10 & $10 \cdot 08(1 \cdot 42)$ \\
Craniospinal irradiation +chemotherapy & 7 & $9 \cdot 41(1 \cdot 44)$ \\
\hline
\end{tabular}

years of age) was above the mid-parental height at final height. All others were short for mid-parental height. This deficit was similar in those who had received either cranial irradiation and chemotherapy or craniospinal irradiation alone, and was most marked in the children who had received both craniospinal irradiation and chemotherapy. The mid-parental height was similar in all four groups.

\section{SEGMENTAL GROWTH DURING PUBERTY Sitting height}

The use of chemotherapy has no apparent detrimental effect on sitting height during pubertal growth. There was a positive correlation between age at irradiation and change in sitting height SD score during puberty in those children who had received craniospinal irradiation, the youngest at irradiation having the greatest reduction in sitting height. As might be expected, cranial irradiation alone did not influence sitting height during puberty.

\section{Leg length}

There was no significant change in leg length SD score during puberty in any of the four treatment groups, that is leg length centile position is maintained by the use of growth hormone.

\section{Duration of puberty}

Table 3 shows the patient numbers, mean, and SD for the length of puberty in each of the four treatment groups as well as the whole population. The numbers in the different groups were small but the different treatment modalities appeared not to affect duration of puberty. The mean length of puberty from G2-G4/B2-B4 in the whole population (1.97 years) is not significantly different from the duration of puberty in normal children (mean 1.8 years in girls and 1.93 years in boys)..$^{13}$

\section{Discussion}

Our results confirm that short term growth velocity is improved with growth hormone treatment in children with irradiation induced growth hormone deficiency. ${ }^{3-5} 18-20$ It is only more recent studies, however, that have distinguished between cranial and craniospinal irradiation. ${ }^{6-9} 21$ Some of these latter studies have included patients with a primary diagnosis of acute leukaemia, as well as brain tumours. Our study has included only those with a primary diagnosis of a brain tumour and hence there are fewer variables in radiation schedules and techniques and chemotherapy protocols. Thirteen patients with a diagnosis of a brain tumour and treated with growth hormone in the earlier studies reported by Clayton et al $^{67}$ form a subset of this current study.

There have been few final height studies in this group of patients. Although Herber et al reported that the growth pattern was not influenced by spinal schedules, ${ }^{21}$ spinal irradiation 
has clearly been shown to have a detrimental impact on final height in the studies by Clayton et $a l^{7}$ and Sulmont et al. ${ }^{8}$ This finding is emphasised in the present report.

The earlier studies did not discuss their results in the context of target genetic height, thereby making it difficult to interpret the magnitude of the growth loss. ${ }^{6-8}$ Our study on larger numbers of patients, and confined only to those children with brain tumours, has analysed for the first time the impact of different components of the initial tumour management on final height. In particular we have assessed the impact chemotherapy has on growth. There are no published data on the influence of chemotherapy on final height. Cytotoxic drugs may impair the production of insulin-like growth factor-1 (IGF-1) by the liver and the action of IGF-1 on the growth plate. ${ }^{22}$ Recently it has been shown that growth in the first four years after treatment with craniospinal irradiation is more profoundly affected in children who have received adjuvant chemotherapy than in those receiving craniospinal irradiation alone, suggesting potentiation of radiation induced growth failure by the chemotherapy. ${ }^{12}$ It has also been suggested that actinomycin- $D$ radiosensitises bone to the effects of radiotherapy, ${ }^{23}$ but it is unknown if other cytotoxic drugs have a similar effect. Although the numbers are small, we have demonstrated that the effect of chemotherapy on final height is as profound as the spinal component of craniospinal irradiation, and the combination of the two treatment modalities causes the most severe growth restriction, the effects being additive. In addition to a direct toxic effect on growing bone, the chemotherapy drugs utilised in the treatment of brain tumours (BiCNU, CCNU) may influence growth by alternative means such as their capacity to induce restrictive lung disease. ${ }^{24}$

Final height is correlated with the age at irradiation, the youngest at irradiation having the worst growth prognosis. As the age at irradiation is inversely correlated with the lag time from irradiation to the start of growth hormone treatment, it may be the latter that is influencing final height. Our treatment criteria have evolved over the years such that all children, irrespective of age, will now be considered for growth hormone treatment if growth hormone deficient two years from diagnosis and radiotherapy. Few of these children thus treated have reached final height so it is not yet possible to analyse the impact of this earlier therapeutic intervention.

Despite the small numbers, it appears there is no effect of chemotherapy either alone or synergistically with cranial irradiation on skeletal proportion at final height (table 4). Those who received craniospinal irradiation were significantly disproportionate but the use of chemotherapy did not exaggerate this disproportion (table 4). The effect of spinal irradiation on spinal growth is not unexpected. Sporadic reports of the effect of irradiation on the growing bones of children have appeared since 1930 with resultant poor growth, underdevelopment, and, when the spine has been irradiated, scoliosis in the affected bone.
Probert et al reported that 10 of 22 children who had received craniospinal irradiation under the age of 15 years had sitting heights greater than 2 SD below the mean. ${ }^{25}$ They suggested that the most vulnerable time for radiation damage to the growing spine was under the age of 6 years and at the time of puberty. The former finding was confirmed by Shalet et al, but they disagreed that spinal irradiation during puberty was particularly likely to impair growth. ${ }^{26} \mathrm{~A}$ conservative estimate of the loss in final height is $9 \mathrm{~cm}$ when spinal irradiation is given at 1 year, $7 \mathrm{~cm}$ when given at 5 years, and $5.5 \mathrm{~cm}$ when given at 10 years. ${ }^{26}$

Clayton and Shalet have shown that spinal growth after irradiation was only modestly restricted in the prepubertal child, but that during puberty, a time when spinal growth is normally a dominant feature of total growth, spinal growth is particularly impaired, resulting in exaggeration of disproportion. ${ }^{27}$ The children irradiated peripubertally had a similar pubertal loss in spinal height to that incurred by the children irradiated prepubertally.

The use of growth hormone in conventional doses does not overcome this radiation induced skeletal dysplasia but it has been suggested that higher doses may do so. ${ }^{28}$ However, the use of 'supraphysiological' doses of growth hormone should be viewed with caution because of its mitogenic potential, ${ }^{29}$ particularly in this population of children treated for cancer.

Darendeliler et al retrospectively studied the pubertal maturation of children with isolated growth hormone deficiency treated with exogenous growth hormone and found that although the onset of puberty was significantly delayed, the duration of puberty between the recording of breast or genitalia stages 2 and 4 was shortened to 1.5 years in both boys and girls. ${ }^{11}$ It was suggested that the synergistic effects of endogenous gonadotrophins and exogenous growth hormone shortens the pubertal process. It has been shown, however, that normal children who enter puberty late have both an attenuated peak growth velocity and duration of puberty. ${ }^{30}$ Therefore it may be that the duration of puberty in these children with idiopathic growth hormone deficiency, rather than being shorter than that seen in normal children, is appropriate for age of pubertal onset. In our population of radiation induced growth hormone deficient children, the duration of puberty in the whole population (1.97 years) was not significantly different from the duration of puberty in Tanner's series of normal children (mean 1.8 years in girls and 1.93 years in boys)..$^{13} 14$ Compared more appropriately, however, with children who enter puberty early and have a compensatory extended duration of puberty, ${ }^{30}$ children with radiation induced early puberty may have a relatively attenuated duration of puberty. There was no significant difference in pubertal duration between the four treatment groups, although the numbers in several of the groups were small. This implies that even after the use of gonadotoxic chemotherapy, pubertal development progresses normally. It has been 
demonstrated that the bone age at the onset of puberty in these children is abnormally early. ${ }^{10}$ Hence bone age should not be used as a predictor of duration of remaining growth potential, as these children progress through bone age maturation abnormally rapidly. It is not yet known if the use of gonadotrophin releasing hormone agonist analogues to halt pubertal progression will improve the final height prognosis in this population.

In conclusion, growth hormone increases growth velocity in children with radiation induced growth hormone deficiency but these children fail to reach their mid-parental height. The use of spinal irradiation and chemotherapy in the original treatment of brain tumours has a marked effect on growth which is not overcome with the use of growth hormone treatment in current doses. Early puberty with a normal duration contributes to poor growth.

We thank Serono Laboratories (UK) Ltd for financial support and Mr David Ryder for help with the statistical analysis.

1 Shalet SM, Beardwell CG, Aarons BM, Pearson D, Morris Jones PH. Growth impairment in children treated for brain tumours. Arch Dis Child 1978; 53: 491-4.

2 Brown IH, Lee TJ, Eden OB, Bullimore JA, Savage DCL. Growth and endocrine function after treatment for medulloblastoma. Arch Dis Child 1983; 58: 722-7.

3 Richards GE, Wara WM, Grumbach MM, Kaplan SL, Sheline GE, Conte FA. Delayed onset of hypopituitarism: Sheline GE, Conte FA. Delayed onset of hypopituitarism: sequelae of therapeutic irradiation of central nervous
system, eye, and middle ear tumors. $₹$ Pediatr 1976; 89: system,

4 Perry-Keene DA, Connelly JF, Young RA, Wettenhall HNB, Martin FIR. Hypothalamic hypopituitarism following external radiotherapy for tumours distant from the adenohypophysis. Clin Endocrinol (Oxf) 1976; 5: 373-80.

5 Shalet SM, Whitehead E, Chapman AJ, Beardwell CG. The effects of growth hormone therapy in children with radiation-induced growth hormone deficiency. Acta Paediatr Scand $1981 ; 70: 81-6$

6 Clayton PE, Shalet SM, Price DA. Growth response to growth hormone therapy follow

7 Clayton PE, Shalet SM, Price DA. Growth response to growth hormone therapy following craniospinal irradiagrowth hormone therapy following crani
tion. Eur f Pediatr 1988; 147: 597-601.

tion. Eur f Pediatr 1988; 147: 597-601. Response to growth hormone treatment and final heigh Response to growth hormone treatment and final height after cranial or craniosp

9 Darendeliler F, Livesey EA, Hindmarsh PC, Brook CGD. Growth and growth hormone secretion in children following treatment of brain tumours with radiotherapy. Acta Paediatr Scand 1990; 79: 950-6.

10 Ogilvy-Stuart AL, Clayton PE, Shalet SM. Cranial irradiation and early puberty. $\mathcal{f}$ Clin Endocrinol Metab 1994; 78: 1282-6.

11 Darendeliler F, Hindmarsh PC, Preece MA, Cox L, Brook CGD. Growth hormone increases rate of puberta maturation. Acta Endocrinol (Copenh) 1990; 122: 414-6.

12 Olshan JS, Gubernick J, Packer RJ, et al. The effects of adjuvant chemotherapy on growth in children with adjuvant chemotherapy on growth in child

13 Marshall WA, Tanner JM. Variations in the pattern of pubertal changes in girls. Arch Dis Child 1969; 44, 291-303.

14 Marshall WA, Tanner JM. Variations in the pattern of pubertal changes in boys. Arch Dis Child 1970; 45: 13-23.

15 Zachertal changes in boys. Arch Dis Child 1970; 45: 13-23. $\mathrm{H}$. Testicular volumes during adolescence. Cross sectional and longitudinal studies. Helvetica Pediatrica Acto 1974; 29: 61-72.

16 Tanner JM, Whitehouse RH, Cameron N, Marshall WA Healy MJR, Goldstein H. Assessment of skeletal maturity and prediction of adult height (TW2 method). London Academic Press, 1983.

17 Tanner JM, Whitehouse RH. Standards for standing height and subischial leg length from birth to maturity: British children 1978. Hertford: Castlemead Publications, 1979.
18 Romshe CA, Zipf WB, Miser A, Miser J, Sotos JF, Newton WA. Evaluation of growth hormone release and cranial irradiation-associated short stature. f Pediatr 1984; 104: $177-81$.

19 Winter RJ, Green OC. Irradiation-induced growth hormone deficiency: blunted growth response and accelerated skeletal maturation to growth hormone therapy. $f$ Pediatr 1985; 106: 609-12.

20 Lannering $\mathrm{B}$, Albertsson-Wikland $\mathrm{K}$. Improved growth response to GH treatment in irradiated children. Acta Paediatr Scand 1989; 78: 562-7.

21 Herber SM, Dunsmore IR, Milner RDG. Final stature in brain tumours other than craniopharyngioma: effect of growth hormone. Horm Res 1985; 22: 63-7.

22 Morris MJ. In vitro effects of anti-leukaemic drugs on cartilage metabolism and their effects on somatomedin production by the liver Manchester: University of Manchester, 1981. (PhD thesis.)

23 Wallace WHB, Shalet SM. Chemotherapy with actinomycin D influences the growth of the spine following mycin D influences the growth of the spine following 20: 177 .

24 O'Driscoll BR, Hasleton PS, Taylor PM, Poulter LW, Gattamaneni HR, Woodcock AA. Active lung fibrosis up to 17 years after chemotherapy with carmustine (BCNU) in childhood. N Engl f Med 1990; 323: 378-82.

25 Probert JC, Parker BR, Kaplan HS. Growth retardation in children after megavoltage irradiation of the spine. Cancer 1973; 32: 634-9.

26 Shalet SM, Gibson B, Swindell R, Pearson D. Effect of spinal irradiation on growth. Arch Dis Child 1987; 62: 461-4.

27 Clayton PE, Shalet SM. The evolution of spinal growth after irradiation. Clin Oncol 1991; 3: 220-2.

28 Sauvion S, Brauner R, Sulmont V, Rappaport R. The effect on growth of increased hGH dosage following cranial (CR) or craniospinal (CS) irradiation. Horm Res 1991; 35 (suppl): 117.

29 Blatt J, Wenger S, Stitely S, Lee PA. Lack of mitogenic effects of growth hormone on human leukemic lymphoeffects of growth hormone on human

30 Bourguignon JP. Variations in duration of pubertal growth: a mechanism compensating for differences in timing of puberty and minimizing their effects on final height. Acta Paediatr Scand 1988; 347 (suppl): 16-24

\section{Appendix}

REGRESSION MODEL

The most general regression model considered allowed for the possibility of completely different linear regressions of final height SD score on age at irradiation (AAI) in each of the four radiotherapy/chemotherapy groups. A sequence of three nested models representing certain simplifications of this full model were fitted to the data and the results summarised in the following table:

\begin{tabular}{|c|c|c|c|}
\hline Model description & $R S S^{\star}$ & $d f$ & $\begin{array}{l}\text { Simplifying } \\
\text { hypothesis }\end{array}$ \\
\hline $\begin{array}{l}\text { Separate regressions } \\
\text { in each of four groups }\end{array}$ & $19 \cdot 07$ & 21 & \\
\hline Parallel regressions & $21 \cdot 75$ & 24 & $\begin{array}{r}F_{3,21}=0.98 \\
(p=0.42)\end{array}$ \\
\hline $\begin{array}{l}\text { Additive effects of chemotherapy } \\
\text { and irradiation }\end{array}$ & $22 \cdot 31$ & 25 & $\begin{array}{r}F_{1,25}=0.63 \\
(p=0.44)\end{array}$ \\
\hline $\begin{array}{l}\text { Equal effects of chemotherapy } \\
\text { and irradiation }\end{array}$ & $22 \cdot 33$ & 26 & $\begin{array}{r}F_{1,24}=0.01 \\
(p=0.82)\end{array}$ \\
\hline
\end{tabular}

$\star R S S=$ residual sum of squares.

The parameter estimates from the last model were:

$\mu=-2 \cdot 895+0.3039 \mathrm{AAI}-1 \cdot 471 \mathrm{X}$

$(0.00569) \quad(0.260)$

$\sigma=0.93$

where $X=0$ cranial irradiation

1 (cranial irradiation plus chemotherapy) or craniospinal irradiation

2 craniospinal irradiation plus chemotherapy 